\title{
Bedsided Transcranial Sonographic Monitoring for Expansion and Progression of Subdural Hematoma Compared to Computed Tomography
}

\author{
Wolf-Dirk Niesen $^{1 *}$, Michael Rosenkranz ${ }^{2}$ and Cornelius Weiller ${ }^{1}$ \\ ${ }^{1}$ Department of Neurology, University Medical Center, University of Freiburg, Freiburg, Germany, ${ }^{2}$ Department of Neurology, \\ Albertinen Hospital Hamburg, Teaching Hospital of the University of Hamburg, Hamburg, Germany
}

Introduction: Transcranial high-resolution ultrasonography reliably allows diagnosis and monitoring of intracerebral hemorrhage in adults. Sonographic monitoring of subdural hematoma (SDH) has not been evaluated in adults so far. This study investigates the reliability of transcranial gray-scale sonography (TGS) in monitoring acute and chronic SDH in adults.

OPEN ACCESS

Edited by:

Barak Bar,

Loyola University Medical Center,

United States

Reviewed by:

Bernhard Schaller,

Universität Zürich, Switzerland

Rick Gill,

Hospital of the University of

Pennsylvania, United States

*Correspondence:

Wolf-Dirk Niesen

wolf-dirk.niesen@uniklinik-freiburg.de

Specialty section:

This article was submitted

to Neurocritical and

Neurohospitalist Care,

a section of the journal

Frontiers in Neurology

Received: 05 January 2018

Accepted: 07 May 2018

Published: 28 May 2018

Citation:

Niesen W-D, Rosenkranz $M$ and

Weiller C (2018) Bedsided

Transcranial Sonographic Monitoring

for Expansion and Progression of

Subdural Hematoma Compared

to Computed Tomography.

Front. Neurol. 9:374.

doi: 10.3389/fneur.2018.00374
Methods: TGS was performed in 47 consecutive patients with either acute or chronic SDH confirmed by cerebral CT. Four patients were excluded due to insufficient bone window. After identification of SDH in TGS extent was measured and correlated with extent of SDH on cerebral computer tomography (CCT). If possible measurement was performed at least on 2 days to evaluate the possibility to monitor SDH with TGS.

Results: In 43 patients with SDH, 76 examinations were performed with 2 examinations in 23 patients and 3 examinations in 10 patients. Overall extent of SDH correlated significantly between TGS and CCT ( $r=0.962)$. Accordingly correlation was high during each single examination time point. In patients in need for surgical evacuation sonographic measurement yielded a sensitivity of $90.9 \%$ and specificity of $93.8 \%$ in predicting surgical evacuation $(p<0.001)$.

Discussion: Imaging of SDH with TGS is possible in patients with SDH and extent of SDH correlates significantly between TGS and CCT during initial as well as during follow-up examination. Thus monitoring of SDH with TGS at patients' bedside is possible.

Keywords: subdural hematoma, neurotrauma, neurocritical care, transcranial sonography, monitoring

\section{INTRODUCTION}

Subdural hematoma (SDH) is a common complication of major traumatic head injury, as well as in recurrent minor head trauma. In case of minor SDH without a sign of mass effect and without clinical symptoms monitoring of SDH without any surgical intervention is feasible (1). Cerebral computer tomography (CCT) currently is the method of choice to monitor SDH (2). Consecutively especially in critically ill patients with limited clinical monitoring serial CCT is performed leading to patient transportation which may place patients at risk. Thus, there is a need for other monitoring techniques that may be applied at patients' bedside.

In infants, transcranial high-resolution ultrasonography through the non-ossified fontanel is the method of choice since it reliably allows diagnosis and monitoring of $\operatorname{SDH}(3,4)$. Studies on 
intracerebral hematomas in adults demonstrated sufficient spatial resolution for diagnosis and measurement of hematoma in transcranial gray-scale sonography (TGS) (5-7) but small cortical hemorrhages could not clearly be distinguished (5). Despite this, SDH may be depicted by TGS (8). Yet, the role of TGS in monitoring $\mathrm{SDH}$ and detecting patients in need for surgical evacuation has not been investigated.

This study evaluates the role of sonographic monitoring of acute and chronic SDH by comparing measurement of SDH expansion between TGS and CCT over time, trying to identify patients in need for surgical evacuation accordingly.

\section{MATERIALS AND METHODS}

47 consecutive patients presenting with acute or chronic SDH were included into the study. Four patients had to be excluded due to insufficient transtemporal bone window.

\section{Inclusion/Exclusion Criteria}

Initial CCT or MRI confirming SDH along the convexity served as the only inclusion criterion. Patients were excluded in other causes of extracerebral space enlargement (e.g., subdural empyema, focal brain atrophy). Also, time interval between TGS and radiologic imaging was not to exceed $12 \mathrm{~h}$ and patients were excluded in case of insufficient transtemporal bone window. Finally, patients were not included if patients or patient relatives did not consent to the study.

\section{Neuroradiological Imaging}

Diagnosis and classification regarding acuity of SDH was achieved by either cerebral CT or cerebral MRI following published criteria. Diagnosis and definition of acute or chronic SDH were as follows: on CCT SDH was diagnosed if CCT revealed either a hyperdense (acute SDH), an isodense (hyperacute SDH), or a hypodense homogenous or inhomogeneous (chronic SDH) crescent-shaped fluid collection along the convexity with a sharp margin to the brain parenchyma. In the few cases with MRIbased diagnosis, SDH was defined as typical crescent-shaped fluid collection along the convexity with typical presentation on T1- and T2-weighed images defining the age of SDH.

Extent of SDH on CCT was measured using the same plane that was used for TGS and included lateral extension, e.g., thickness of hematoma only. Measurement was performed by an experienced neuroradiologist. Follow-up CCT examination was performed according to clinical necessity and published clinical procedural criteria.

\section{Transcranial Gray-Scale Sonography}

Sonographic examination was performed using a color-flow ultrasound system feasible for transcranial color-coded duplex sonography (Siemens Sonoline Elegra, Siemens AG, Erlangen; Sonos 5500, Philips, Hamburg) using a $2.0-2.5 \mathrm{MHz}$ phasedarray transducer. For insonation, the transtemporal bone window was used in an axial plane, and evaluation of brain parenchyma was performed according to published criteria $(9,10)$. SDH was visualized with a scanning depth of $16 \mathrm{~cm}$ and with progression from the side opposite to the pathology confirmed by CCT. The investigator was notified concerning existence and localization of the SDH but was blinded to extent of SDH. Demonstration of a highly echogenic membrane clearly distinctable from the echogenic opposing skull was defined as the dural border of the arachnoid and as a sign of subdural space enlargement on transcranial gray-scale imaging (Figure 1). Extent of subdural space enlargement was measured by drawing a perpendicular line from the opposing skull to the inside of the echogenic membrane at the broadest part of subdural space enlargement. Sonographic measurement was performed within $12 \mathrm{~h}$ of corresponding CCT examinations.

\section{Statistics}

After testing for equal distribution using the Shapiro-Wilk test overall extent of SDH on TGS was correlated with extent on CCT using Pearson's correlation coefficient. Also, extent of SDH of the single examination was correlated between TGS and CCT using Pearson's correlation coefficient. Also, the rate of progressing $\mathrm{SDH}$ with a need for surgical evacuation was calculated from TGS and sensitivity as well specificity was calculated by ROC analysis. Statistical analysis was performed using SPSS statistical software (SPSS Version 17.0).

\section{Ethics}

This study was carried out in accordance with the recommendations of the ethics committee of Hamburg University and Freiburg University, Germany, with written informed consent from all subjects or their legal guardians. All subjects gave written informed consent in accordance with the Declaration of Helsinki. The protocol was approved by the ethics committee of Freiburg University, Germany.

\section{RESULTS}

47 consecutive patients with SDH confirmed by CCT were included into the study (see Table 1). TGS was performed within $12 \mathrm{~h}$ of CCT in all patients. Four patients presented

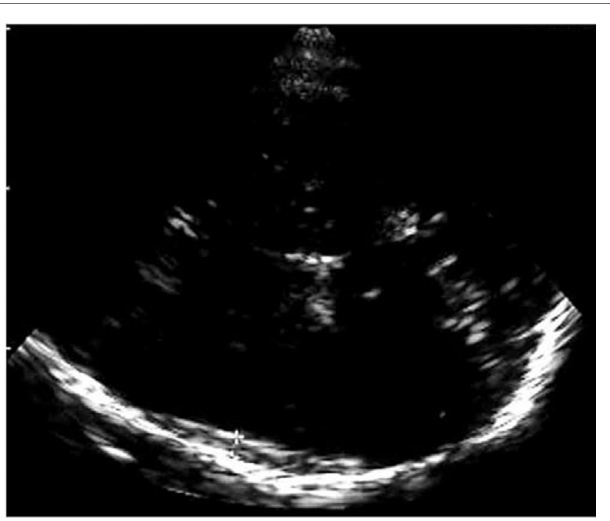

FIGURE 1 | Figure shows the highly echogenic skull opposite to the probe and a highly echogenic membrane clearly distinctable from the skull as sonographic correlate of the dural border of the arachnoid and as a sign of subdural space enlargement. 
TABLE 1 | Patients' characteristics.

\begin{tabular}{lcc}
\hline & $\boldsymbol{n}$ & Percentage \\
\hline All patients & 47 & 8.5 \\
Insufficient transtemporal bone window & 4 & \\
Included patients & 43 & \\
Examinations overall & 76 & 53.5 \\
1 follow-up & 23 & 23.3 \\
2 follow-ups & 10 & \\
Age (mean \pm SD) & 16.4 years & 76.7 \\
Male & 33 & 23.3 \\
Female & 10 & \\
Acuity of subdural hematoma & & 76.7 \\
Acute & 33 & 11.6 \\
$\quad$ chronic & 5 & 11.6 \\
$\quad$ Acute on chronic & 5 & 25.6
\end{tabular}

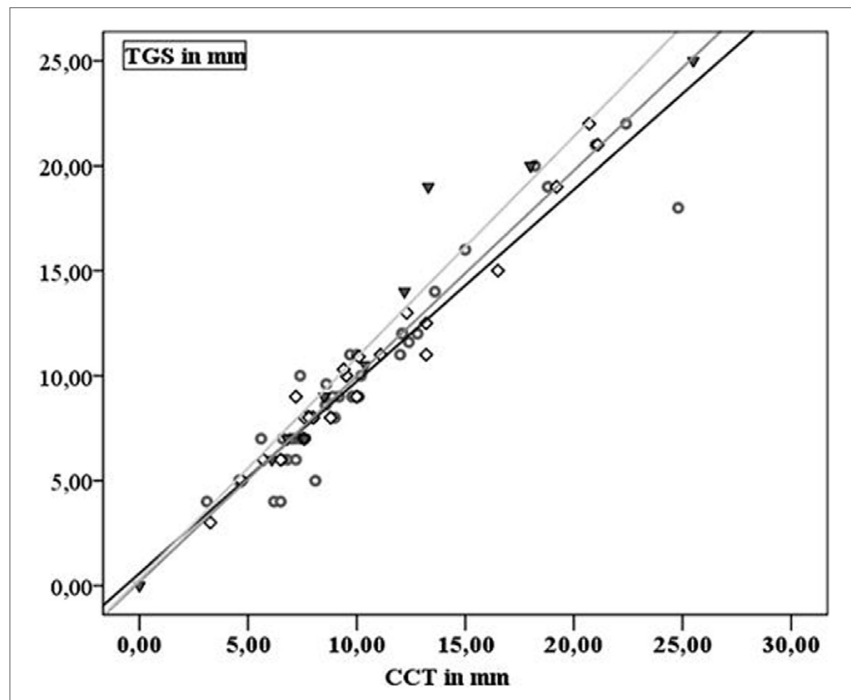

FIGURE 2 | Gray dots and black line: first examination; black squares and dark gray line: second examination; black triangles and gray line: third examination.

without a transtemporal bone window and were excluded from the study. SDH could be visualized in 43 patients (91.5\%). 38 patients presented with acute SDH and 5 with purely chronic SDH.

Extent of SDH was measured in 43 patients and ranged from 4 to $25 \mathrm{~mm}$ on CCT and from 3.1 to $25.5 \mathrm{~mm}$ on TGS. In 23 patients, 1 follow-up examination by TGS and CCT was performed and 10 patients received a second follow-up. In 76 examinations performed SDH extension demonstrated a highly positive linear correlation between TGS and CCT $(r=0.962$; $p<0.001)$. Looking at the different examination time points TGS and CCT demonstrated positive linear correlation at al time points (initial examination: $r=0.954, p<0.001$; first follow-up examination: $r=0.983, p<0.001$; second follow-up examination: $r=0.97, p<0.001$ ) (Figure 2). In 11 patients, acute evacuation of SDH due to SDH expansion was necessary. Sonographic measurement had a sensitivity of $90.9 \%$ and a specificity of $93.8 \%$ in

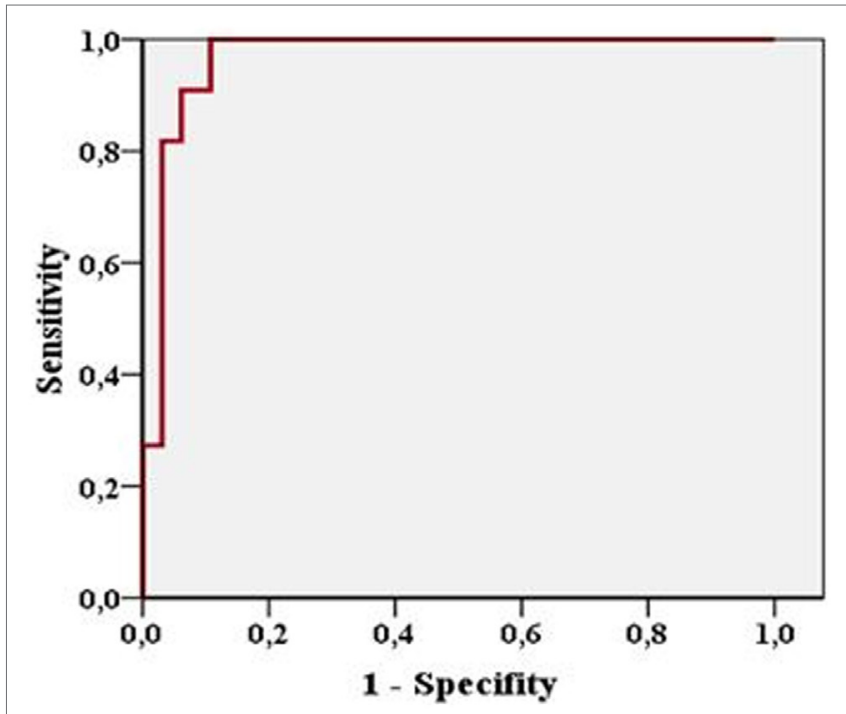

FIGURE 3 | Figure shows high sensitivity and specificity of sonographic measurement in predicting surgical evacuation of subdural hematoma.

predicting surgical intervention when sonographic extension of SDH exceeded above $13.25 \mathrm{~mm}(p<0.001)$ (Figure 3).

\section{DISCUSSION}

Transcranial gray-scale sonography is the method of choice to diagnose and monitor extracerebral space enlargement in newborn and infants. In addition, distinction of benign subarachnoid space enlargement, hydrocephalus externus, bacterial meningitis, and SDH and hygroma is possible $(4,11)$. Differentiation of the subarachnoid and the subdural space is based on the depiction of vessels within the subarachnoid space and the depiction of an echogenic membrane. The echogenic membrane is the sonographic correlate of the dural border of the arachnoid, separating the subarachnoid from the subdural space $(4,11,12)$. Also, some authors propagate intraoperative ultrasound in SDH evacuation to detect deep-seated isolated hematomas in need for fenestration during surgery (13) as well as complicating contralateral epidural or SDHs in decompressive surgery (14). Based on pediatric ultrasound experience, it has been demonstrated that visualization of SDH in adults is possible even in poor transtemporal bone window. Also, differentiation of acute and chronic SDH is possible and correlation of extension of SDH between CCT and TGS is high (8).

Treatment of SDH usually is surgical but conservative treatment is feasible in patients with small SDH extension and no sign of mass effect (1). Especially in chronic and partially acute on chronic SDH spontaneous resorption and consecutive regression have been described $(15,16)$. Criteria that qualify for conservative treatment are clinical as well as radiological (1). So far sequential CCT monitoring is the method of choice in monitoring SDH besides clinical evaluation (2). It has been shown that in some patients with traumatic head injuries, who have not undergone surgical intervention in the beginning, changes on serial CCT 
may precede clinical deterioration especially in extending SDH (17). In addition, changes on CCT with or without clinical deterioration are associated with a worse outcome thus indicating serial imaging (18). Despite this, CCT is a radiological method requiring patient transportation to the radiology suite and placing the patients at risk during transportation. Thus, there is a need for noninvasive bedside monitoring of conservatively treated SDH.

With the presented study we were able to show that correlation of TGS measured extension of SDH with CCT is high during the time course of SDH monitoring. In case of SDH expansion on CCT TGS detected expansion of SDH as well. These data are in accordance with data from pediatric ultrasound $(11,19,20)$. In addition, these findings are in accordance with data from studies comparing the value of transcranial monitoring of intraparenchymal hematoma with CCT. In several studies, monitoring of intracerebral hematoma with ultrasound was possible and size, volume, and localization of intracerebral hemorrhage measured by TGS are highly acurate compared with CCT $(5-7,21)$. It was even possible to differentiate the different phases of hematoma resorption via TGS $(5,7)$. Although we did not monitor for changes of echogenicity of SDH, we were able to demonstrate that it is possible to monitor extension of SDH with TGS since correlation of TGS und CCT measurement of SDH extent remained high during follow-up. In accordance with a reliable detection rate of intracerebral hematoma volume expansion in several studies $(6,7)$, we were also able to clearly identify extensive SDH and $\mathrm{SDH}$ enlargement in need for surgical evacuation either primarily or during follow-up with a high sensitivity and specificity. Therefore, even though CCT is necessary for the surgical intervention, monitoring SDH may be performed with TGS up to this point since TGS shows reliable detection of patients in need for surgical evacuation of SDH. By adding the sonographic measurement of midline-shift comparable to studies on mass effect due to intracerebral hematoma or malignant stroke $(22,23)$ may allow an even closer monitoring of SDH expansion (24).

Our study on sonographic monitoring has some limitations that need to be addressed. Results are limited by the relatively small number of patients and follow-up examinations thus revealing progression of hematoma in need for surgical evacuation in a limited number of patients only. This might have influenced the high correlation between sonographically derived need for surgery and the clinical and radiological based indication for evacuation of hematoma. In addition, we did not monitor for other signs of hematoma-associated mass effects other than SDH extension, e.g., edema formation with midline shift. Consecutively, smaller $\mathrm{SDH}$ in need for surgical intervention due to progressive edema

\section{REFERENCES}

1. Wong CW. Criteria for conservative treatment of supratentorial acute subdural haematomas. Acta Neurochir (Wien) (1995) 135(1-2):38-43. doi:10.1007/ BF02307412

2. Besenski N. Traumatic injuries: imaging of head injury. Eur Radiol (2002) 12(6):1237-52. doi:10.1007/s00330-002-1355-9

3. Huang CC, Shen EY. Tentorial subdural hemorrhage in term newborns: ultrasonographic diagnosis and clinical correlates. Pediatr Neurol (1991) 7(3):171-7. doi:10.1016/0887-8994(91)90080-5 formation would have been missed. Therefore, further studies on combining sonographic parameters such as midline shift and compression of the ventricular system with TGS monitoring of SDH extension are needed in a larger set of patients for a more precise detection of SDH in need for surgery. Transcranial sonography and consecutively sonographic monitoring of SDH is limited due to an insufficient transtemporal bone window. Also, SDH localized in the posterior fossa or highly fronto-parietal and $\mathrm{SDH}$ progression within this region may not be detected due to the anatomy of the transtemporal bone window. In our study, SDH could not be depicted in $8.5 \%$ patients only. Furthermore, TGS is an investigator-dependent method and reliability of results relies on the experience of the investigator. Finally, the presented study describes the role of TGS in monitoring SDH diagnosed either by CCT or MRI. Thus, TGS may only serve as a monitoring tool at patients' bedside, while the possibility of primary detection of SDH with TGS as described in infants remains unknown.

To summarize our results, we were able to show that monitoring of SDH with TGS is possible. TGS reliably identifies patients that may be in need for surgical evacuation of SDH, even though we did not monitor for secondary change due to SDH, e.g., ventricular compression or midline shift. Thus, TGS is an intriguing monitoring method especially in the critically ill since TGS may be performed at the patients' bedside. Measurement of SDH via TGS is impossible in patients without transtemporal bone window only and thus may be performed in a high percentage of patients with SDH reducing transportation of critically ill patients as well as radiation strain due to serial CCT scanning.

\section{ETHICS STATEMENT}

This study was carried out in accordance with the recommendations of the ethics committee of Hamburg University and Freiburg University, Germany with informed consent in accordance with the Declaration of Helsinki of all subjects or their legal guardians.

\section{AUTHOR CONTRIBUTIONS}

W-DN has carried out the investigation (acquisition of clinical, radiological, and sonographical data) described in the study and has drafted the manuscript as well as the figures submitted and thus mainly has contributed to the work presented. MR has performed part of the data acquisition (sonographic examination) and has added substantial revision to the manuscript. CW contributed to the conception and design of the study and has contributed substantial revision to the manuscript.
4. Rupprecht T, Lauffer K, Storr U, Hofbeck M, Wenzel D, Böwing B. Extra-cerebral intracranial fluid collections in childhood: differentiation between benign subarachnoid space enlargement and subdural effusion using color-coded duplex sonography. Klin Padiatr (1996) 208(3):97-102. doi:10.1055/s-2008-1044003

5. Seidel G, Kaps M, Dorndorf W. Transcranial color-coded duplex sonography of intracerebral hematomas in adults. Stroke (1993) 24:1519-27. doi:10.1161/ 01.STR.24.10.1519

6. Matsumoto N, Kimura K, Iguchi Y, Aoki J. Evaluation of cerebral haemorrhage volume using transcranial color-coded duplex sonography. J Neuroimaging (2011) 21(4):355-8. doi:10.1111/j.1552-6569.2010.00559.x 
7. Perez ES, Delgado-Mederos R, Rubiera M, Delgado P, Ribo M, Maisterra O, et al. Transcranial duplex sonography for monitoring hyperacute intracerebral haemorrhage. Stroke (2009) 40(3):987-90. doi:10.1161/STROKEAHA. 108.524249

8. Niesen WD, Burkhardt D, Hoeltje J, Rosenkranz M, Weiller C, Sliwka U. Transcranial grey-scale sonography of subdural haematoma in adults. Ultraschall Med (2006) 27(3):251-5. doi:10.1055/s-2006-926544

9. Berland LL, Bryan CR, Sekar BC, Moss CN. Sonographic examination of the adult brain. JClin Ultrasound (1988) 16:337-45. doi:10.1002/jcu. 1870160508

10. Bogdahn U, Becker G, Winkler J, Greiner K, Perez J, Meurers B. Transcranial color-coded real-time sonography in adults. Stroke (1990) 21:1680-8. doi:10.1161/01.STR.21.12.1680

11. Libicher M, Rohrschneider W, Troger J, Roeren T, Kauffmann GW. Ultrasound of extracerebral spaces in infants: standardized examination technique. Ultraschall Med (1996) 17(6):281-4. doi:10.1055/s-2007-1003199

12. Veyrac C, Couture A, Baud C. Pericerebral fluid collections and ultrasound. Pediatr Radiol (1990) 20(4):236-40. doi:10.1007/BF02019655

13. Shimizu S, Mochizuki T, Osawa S, Kumabe T. Intraoperative ultrasonography during drainage for chronic subdural hematomas: a technique to release isolated DEPP-seated hematomas - technical note. Neurol Med Chir (Tokyo) (2015) 55:781-5. doi:10.2176/nmc.tn.2015-0074

14. Huang AP, Chen YC, Hu CK, Lin TK, Huang SJ, Tu YK, et al. Intraoperative sonography for detection of contralateral acute epidural or subdural hematoma after decompressive surgery. J Trauma (2011) 70(6):1578-9. doi:10.1097/ TA.0b013e3182178c71

15. Parlato C, Guarracino A, Moraci A. Spontaneous resolution of chronic subdural hematoma. Surg Neurol (2000) 53(4):312-5. doi:10.1016/S00903019(00)00200-7

16. Servadei F, Nasi MT, Giuliani G, Cremonini AM, Cenni P, Zappi D, et al. CT prognostic factors in acute subdural haematomas: the value of the 'worst' CT scan. Br J Neurosurg (2000) 14(2):110-6. doi:10.1080/02688690050004525

17. Bee TK, Magnotti LJ, Croce MA, Maish GO, Minard G, Schroeppel TJ, et al. Necessity of repeat head CT and ICU monitoring in patients with minimal brain injury. J Trauma (2009) 66(4):1015-8. doi:10.1097/ TA.0b013e31819adbc8

18. Thorson CM, Van Haren RM, Otero CA, Guarch GA, Curia E, Barrera JM, et al. Repeat head computed tomography after minimal brain injury identifies the need for craniotomy in the absence of neurologic change. J Trauma Acute Care Surg (2013) 74(4):967-73. doi:10.1097/TA.0b013e3182877fed

19. Naidich TP, Yousefzadeh DK, Gusnard DA. Sonography of the normal neonatal head. Supratentorial structures: state-of-the-art imaging. Neuroradiology (1986) 28:408-27. doi:10.1007/BF00344096

20. Libicher M, Tröger J. US measurement of the subarachnoid space in infants: normal values. Radiology (1992) 184:749-51. doi:10.1148/radiology.184. 3.1509061

21. Woydt M, Greiner K, Perez J, Becker G, Krone A, Roosen K. Transcranial duplex-sonography in intracranial hemorrhage. Evaluation of transcranial duplex-sonography in the diagnosis of spontaneous and traumatic intracranial hemorrhage. Zentralbl Neurochir (1996) 57(3):129-35.

22. Tang SC, Huang SJ, Jeng JS, Yip PK. Third ventricle midline shift due to spontaneous supratentorial intracerebral hemorrhage evaluated by transcranial color-coded sonography. J Ultrasound Med (2006) 25(2):203-9. doi:10.7863/ jum.2006.25.2.203

23. Stolz E, Gerriets T, Fiss I, Babacan SS, Seidel G, Kaps M. Comparison of transcranial color-coded duplex sonography and cranial CT measurements for determining third ventricle midline shift in space-occupying stroke. AJNR Am J Neuroradiol (1999) 20(8):1567-71.

24. Cattalani A, Grasso VM, Vitali M, Gallesio I, Magrassi L, Barbanera A. Transcranial color-coded duplex sonography for evaluation of midline-shift after chronic-subdural hematoma evacuation (TEMASE): a prospective study. Clin Neurol Neurosurg (2017) 162:101-7. doi:10.1016/j.clineuro.2017.09.015

Conflict of Interest Statement: None of the authors received any financial compensation for their contribution to this study. W-DN has received travel expense funding of Fresenius respectively with no potential conflict of interest. MR has no relationships/conditions/circumstances that present potential conflict of interest. $\mathrm{CW}$ in the past has received payment for consultancy for Pierre Fabre with no potential conflict of interest.

Copyright (c) 2018 Niesen, Rosenkranz and Weiller. This is an open-access article distributed under the terms of the Creative Commons Attribution License (CC BY). The use, distribution or reproduction in other forums is permitted, provided the original author(s) and the copyright owner are credited and that the original publication in this journal is cited, in accordance with accepted academic practice. No use, distribution or reproduction is permitted which does not comply with these terms. 